\title{
Thermal conductivity of phononic membranes with aligned and staggered lattices of holes at room and low temperatures
}

\author{
Maxime Verdier, ${ }^{1, *}$ Roman Anufriev, ${ }^{2}$ Aymeric Ramiere, ${ }^{2,3}$ Konstantinos Termentzidis, ${ }^{1}$ and David Lacroix ${ }^{1}$ \\ ${ }^{1}$ Université de Lorraine, LEMTA-CNRS, UMR 7563, Vandoeuvre-lès-Nancy, F-54500, France \\ ${ }^{2}$ Institute of Industrial Science, The University of Tokyo, 153-8505 Tokyo, Japan \\ ${ }^{3}$ LIMMS/CNRS-IIS, UMI 2820, The University of Tokyo, 153-8505 Tokyo, Japan \\ (Received 24 March 2017; revised manuscript received 3 May 2017; published 30 May 2017)
}

\begin{abstract}
The in-plane thermal conductivity of silicon phononic membranes is investigated by micro time domain thermoreflectance and Monte Carlo simulations. Strong reduction of thermal conductivity is observed mainly due to phonon boundary scattering for both aligned and staggered lattices of holes. The measured and calculated thermal conductivities of the porous membranes with cylindrical holes are found to be in good quantitative agreement (at $4 \mathrm{~K}$ and $300 \mathrm{~K}$ ). A significant difference between thermal conductivities of aligned and staggered lattice of identical porosities is observed. This difference is shown to arise from ballistic phonons that acquired directionality by propagating between the holes. The directionality effect strengthens when the temperature is decreased or when the diameter of the holes becomes close to the period. Finally, we propose a model, which quantifies and explains the difference between thermal conductivities of aligned and staggered lattices based on geometric considerations.
\end{abstract}

DOI: 10.1103/PhysRevB.95.205438

\section{INTRODUCTION}

Physics of nanoscale heat conduction has attracted high attention due to its importance for various applications in the fields of microelectronics, optoelectronics, and energy harvesting, but also for its intriguing divergence from classical physics of heat conduction [1]. This is particularly true for dielectrics and semiconductors where heat is mostly carried by phonons (quanta of normal modes resulting from lattice vibrations). Unlike in bulk, heat conduction in nanostructures cannot always be described by Fourier's law, as these phonons are scattered by the boundaries of the structure, which can significantly reduce thermal conductivity. As a consequence, thermal transport at length scales comparable to the phonon mean free paths (MFPs) or to the phonon wavelengths is now a topic of great interest.

In the past few years, reduced thermal conductivity has been demonstrated in various semiconductor nanostructures, like thin nanofilms [2,3], nanowires [4,5], nanoporous materials [6,7], and, more recently, thin membranes with periodic arrays of holes [8-11] known as phononic membranes. The latter nanostructures are very promising for controlling heat transport without affecting the electron transport properties much, thus favoring potential applications in thermoelectricity [10]. In phononic membranes, the thermal conductivity reduction is mostly caused by the phonon scattering on hole boundaries $[8,12,13]$ and thus correlates with the surface-to-volume ratio of the structure $[8,14,15]$. Recent experiments $[8,10]$ have also indicated that thermal conductivity of complex nanostructures, such as phononic membranes might be controlled solely by the smallest dimensions of the structure, as the narrowest regions naturally limit the phonon total MFP $[13,16]$. However, despite these advances, characteristic lengths at which these nanoscale phenomena take place remain unclear, and complete understanding of heat conduction in phononic membranes requires a systematic experimental and theoretical investigation

\footnotetext{
*Corresponding author: maxime.verdier@univ-lorraine.fr
}

of phonon transport in structures of different sizes and for different lattices of holes (aligned, staggered, hexagonal, etc.).

In this paper, we investigate the in-plane thermal conductivity in silicon phononic membranes of different periods and lattices using micro-time domain thermoreflectance ( $\mu$-TDTR) measurements and Monte Carlo (MC) simulations. We find good quantitative agreement between experiments and simulations showing that thermal conductivity of phononic membranes can be explained by limitations of total MFPs caused by phonon scattering on the hole boundaries. Moreover, our low-temperature simulations indicate the presence of ballistic phonon transport, which shows heat directionality between the holes of phononic membranes.

The paper is organized as follows: the successive steps about elaboration of phononic membranes and how their thermal properties are measured using $\mu$-TDTR are briefly detailed in Sec. II; then, the Monte Carlo method used to solve the Boltzmann Transport Equation is described in Sec. III. Particular attention is paid to the description of characteristic phonon MFPs due to three-phonon processes and boundary scattering. Experimental and simulation results are given in section IV; thermal conductivity for both aligned and staggered phononic membrane lattices is investigated, and its variation as a function of phonon MFPs is discussed, including accumulated thermal conductivity considerations. Section $\mathrm{V}$ is focused on the impact of hole lattice in phononic membranes at low and room temperature. Ballistic/diffusive transport is studied, and a model based on a "direct passage" theory is suggested to understand the impact of lattice, periodicity/size of holes, on thermal conductivity lowering. A brief conclusion summarizes the main outputs of this paper, and appendixes support our arguments.

\section{SAMPLE PREPARATION AND THERMAL CONDUCTIVITY MEASUREMENTS}

\section{A. Sample fabrication}

The phononic membrane samples were fabricated on a silicon-on-insulator wafer with $1-\mu$ m-thick buried $\mathrm{SiO}_{2}$ layer 

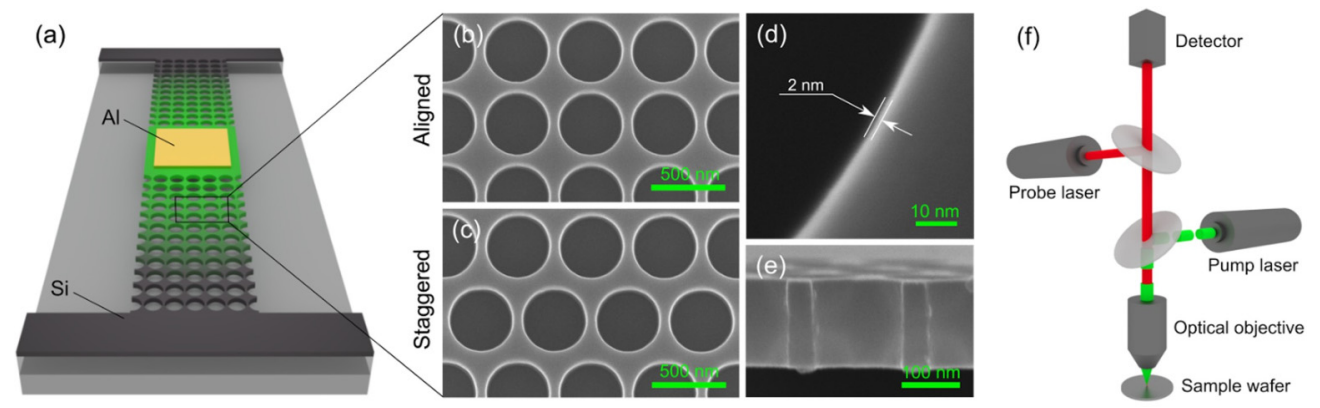

FIG. 1. (a) Schematic of a typical sample and SEM images of phononic membranes with (b) aligned and (c) staggered lattices of holes. Scale bars are $500 \mathrm{~nm}$. (d) High-resolution SEM image of a hole surface showing that surface roughness does not exceed $2 \mathrm{~nm}$. Scale bar $=10 \mathrm{~nm}$. (e) Cross-sectional SEM image showing vertical hole profiles. (f) Simplified schematic of $\mu$-TDTR experimental setup.

and a 145-nm-thick top monocrystalline (100) silicon layer. First, we deposited 125-nm-thick aluminum pads on the top of the silicon layer using electron beam physical vapor deposition. Next, the phononic membranes were formed in the silicon layer using electron beam lithography followed by dry reactive ion etching. Finally, the structures were suspended using hydrofluoric vapor that removed the buried $\mathrm{SiO}_{2}$ layer. Thus, each sample consisted of a 5- $\mu \mathrm{m}$-wide, $25-\mu \mathrm{m}$-long, and 145-nm-thick suspended silicon bridge, $4 \times 4 \mu \mathrm{m}$ [2] aluminum pad in the center of the bridge, and phononic membrane regions on both sides of the bridge, as shown in Fig. 1(a).

To investigate how hole alignment affects heat conduction in phononic membranes, we compared samples with aligned [Fig. 1(b)] and staggered [Fig. 1(c)] arrays of holes. The staggered lattice is formed by shifting every second row of the aligned square lattice by half a period and should not be confused with a hexagonal lattice, as both aligned and staggered lattices have the same porosity, density of holes, and surface-to-volume ratio. To study the heat conduction at different characteristic lengths, we fabricated samples with three different periods ( $a=200,350$, and $500 \mathrm{~nm}$ ) and several different porosities for each period. Two identical sets of samples were fabricated.

To evaluate the quality of samples, we performed atomic force microscopy (AFM) on the top surface of the wafer and high-resolution scanning electron microscopy (SEM) on the hole boundaries. The AFM analysis showed an atomically smooth top surface with surface roughness well below $0.5 \mathrm{~nm}$. Although surface roughness of holes cannot be precisely measured by the SEM, we estimated that the roughness is less than $2 \mathrm{~nm}$, as shown in Fig. 1(d). Figure 1(e) shows vertical hole profiles. Since all samples of the same set were fabricated simultaneously on the same wafer, we do not expect variations of surface roughness from one sample to another.

\section{B. $\mu$-TDTR experimental technique}

To study heat conduction in our samples we used the $\mu$-TDTR technique. Figure 1(f) shows a simplified schematic of the setup. Pulsed pump laser $(\lambda=642 \mathrm{~nm})$ and continuous wave probe laser $(\lambda=785 \mathrm{~nm})$ were focused by an optical objective $(\times 40)$ on samples placed in a He-flow cryostat under high vacuum $\left(<10^{-4} \mathrm{~Pa}\right)$. At each temperature, we used the minimum possible power of the lasers, so that the laser beams do not increase the temperature of samples by more than few kelvins. Every $100 \mu \mathrm{s}$, the pump laser heated the metal pad with a $1-\mu$ s-long pulse, while the probe laser measured subsequent changes in the reflectance $\Delta R / R$, related to a change in temperature, of the metal pad. A typical TDTR signal consisted of a quick rise caused by the pump pulse and exponential decay due to heat dissipation from the metal pad.

To extract the thermal conductivity of phononic membranes at room temperature, we modeled the experiment with the same sample geometry, including holes but without roughness, using the finite element method (FEM) implemented by Comsol Multiphysics 4.1. In the FEM model, we used thermal conductivity of the phononic membrane regions as a free parameter; thus, once the simulated signal matched the experimental TDTR signal, we obtained the thermal conductivity of the phononic membrane.

To minimize the measurement inaccuracy, each sample was measured twice on different days. Thus, for each type of phononic membrane we obtained four values of thermal conductivity (i.e., two values on two identical sets of samples). The presented values of thermal conductivity at room temperature are the average of these four measurements with error bars showing the standard deviation. More details on our experimental technique and modeling can be found in Ref. [16].

Additionally, to gain some insight into the low temperature physics of phononic membranes, we measured one set of samples at $4 \mathrm{~K}$. Since at $4 \mathrm{~K}$ we will consider only relative values of thermal conductivity, we used simplified FEM simulations in which we simulated a membrane without holes to extract the effective thermal conductivity $\left(\kappa_{\text {eff }}\right)$ of each sample. The effective thermal conductivity is related to the regular thermal conductivity as $\kappa=\kappa_{\text {eff }} / F$, where $F$ is the volume correction factor, which is canceled when the ratio of thermal conductivities of aligned and staggered samples is considered. This simplified approach is much less time consuming and generates a smaller additional inaccuracy caused by the FEM modeling.

\section{MODELING PHONON TRANSPORT IN PHONONIC MEMBRANES}

\section{A. Monte Carlo solution of BTE in phononic membranes}

The Boltzmann Transport Equation (BTE) for phonons is solved with a MC technique in the relaxation time 


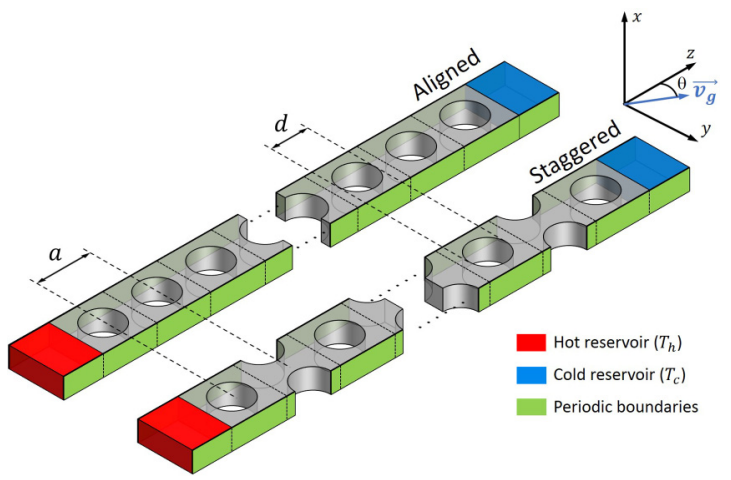

FIG. 2. Schematic representation of the aligned and staggered phononic membranes for MC simulations, where $a$ is the period and $d$ is the diameter of the holes.

approximation [Eq. (1)]. The phonon's distribution function $f$ at a given temperature is derived from the tracking of pseudoparticles that carry a given energy $\hbar \omega$ and move with a group velocity given by $\boldsymbol{v}_{g}=\nabla_{\boldsymbol{K}} \omega$. These properties may change during the simulated time if the pseudoparticles interact via phonon-phonon processes, scattering on impurities and scattering on the geometrical boundaries of the nanostructure. Such an approach, formerly developed for bulk and thin films [17], was improved using a reduced variance technique [18] and successfully adapted to porous media [19].

$$
\frac{\partial f}{\partial t}+\nabla_{\boldsymbol{K}} \omega \cdot \nabla_{\boldsymbol{r}} f=\frac{f^{0}-f}{\tau(\omega, p, T)}
$$

In Eq. (1), $\tau$ is the relaxation time, while $f^{0}$ is the BoseEinstein distribution at thermal equilibrium. The main features of the MC method applied to the BTE are extensively detailed in previous studies [17-19]. We just recall here the successive steps to model heat transport by phonons in a nanostructure like a phononic membrane:

(1) Design of the geometry of the phononic membrane where characteristic lengths and boundary conditions are defined. Here cylindrical holes are set with axis along the $x$ direction (see Fig. 2). They can be in an aligned or a staggered configuration.

(2) Discretization of the system, which is divided into 20 cells of one period length along the $z$ direction. Periodic conditions are set along the $y$ direction. For the top and bottom surfaces of the membrane (along the $x$ direction), boundary conditions are set as diffuse at $300 \mathrm{~K}$ and specular at $4 \mathrm{~K}$ considering the phonon wavelengths at these temperatures. Reflections at the holes are always set as specular (see Appendix A for a justification of specular reflections at the holes at room temperature). The first and the last cells are blackbodies, playing the roles of hot and cold reservoirs, where the temperature is kept constant in order to create a thermal gradient within the structure.

(3) Sampling of phonon population in each cell (i.e., frequency, polarization, and group velocity, using an energy cumulative distribution function [19]). For each phonon, random space position and propagation direction are assigned during this stage. Phonon spectral properties of Si (BoseEinstein distribution, dispersion curves, and density of states) are derived from bulk crystalline characteristics using data provided by Pop et al. [20].

(4) Advective transport of phonons with respect to their initial positions, propagation directions, and simulation time step $(\delta t)$. Reflections at geometrical boundaries are calculated during this step.

(5) Internal phonon scattering, which occurs after the drift phase in order to restore thermal equilibrium. The scattering probability is calculated taking into account the phonon relaxation time $\tau(\omega, p, T)$ as $P_{\text {scat }}=1-\exp (-\delta t / \tau)$, and random numbers are drawn to decide if umklapp, normal, or impurity scattering occurs [17]. According to the considered phenomenon, frequency, polarization, group velocity, and/or propagation directions are resampled. In this paper semiempirical lifetimes proposed by Holland are used [21]. Constants used in the latter phonon lifetime expressions are adjusted to take into account realistic dispersion properties and to recover bulk thermal conductivity of silicon at both low and room temperatures. Those parameters are given in the appendix of a previous paper [19].

(6) Thermalization of the first and last cells according to the prescribed temperature gradient. Calculation of the phonon heat flux $J_{z}$ is achieved considering the energy transported by all the sampled phonons in the $z$ direction: $J_{z}=1 / V \times \sum \hbar \omega \boldsymbol{v}_{g, z}$, where $V$ is the volume of a simulation cell.

During a MC simulation, the last three steps are repeated until steady state (constant heat flux) is reached. Simulation time step is typically set to $1 \mathrm{ps}$. For the considered phononic membranes with lengths ranging from 3.2 to $10 \mu \mathrm{m}$, total duration of 200 to 800 ns were respectively considered to ensure that steady state is reached. Besides, to lower uncertainties when calculating the thermal conductivity due to heat flux fluctuations, simulations are parallelized on 16 cores. Finally, temperatures and fluxes are averaged over the steady-state regime, and the thermal conductivity $(\kappa)$ is extracted according to the Fourier's law $J_{z}=-\kappa \nabla T$.

\section{B. Models and postprocessing}

During $\mathrm{MC}$ simulations, the temperature and heat flux profiles along the temperature gradient are recorded in order to compute the thermal conductivity. Additionally, simulations can provide information on the thermal conductivity dependence on the MFP (intrinsic and geometrical) and the phonons propagation directions in phononic membranes with different lattices.

\section{Thermal conductivity as a function of carrier mean free path}

Since the phonon dispersion and lifetimes inputs of the MC procedure are frequency dependent, the thermal conductivity accumulation as a function of frequency or three-phonon MFP can be extracted from the sampling of the heat flux on spectral intervals. For this calculation, the average spectral intrinsic MFP $\Lambda(\omega)$ is considered in the frame of three-phonon normal and umklapp processes using Holland's relaxation times [22]; it reads

$$
\frac{1}{\Lambda(\omega, T)}=\frac{1}{3}\left(\frac{2}{\Lambda_{\mathrm{TA}}(\omega, T)}+\frac{1}{\Lambda_{\mathrm{LA}}(\omega, T)}\right)
$$


where $\Lambda_{p}(\omega, T)=v_{g}(\omega, p) \times \tau(\omega, p, T)$, with $p$ corresponding to the transverse acoustic (TA) and longitudinal acoustic (LA) polarizations. Using this spectral three-phonon MFP, modes that mainly contribute to heat conduction can be distinguished. A reference analysis is done for bulk silicon and then compared with thin-film and phononic membranes in order to see how material downsizing affects the thermal properties.

In nanostructures, multiple scattering events on the geometrical boundaries lower the thermal conductivity compared with bulk. This additional scattering phenomenon can be characterized by a boundary scattering MFP $\Lambda_{\text {scat bound }}$. As detailed in Ref. [23], a ray tracing technique can be easily implemented in the MC simulation tool used to solve the BTE. Within a single time step, a large number of phonons $N_{\mathrm{ph}}$ are randomly drawn within the nanostructure and allowed to drift with respect to their group velocities. Some of them will collide at the boundaries (walls or holes), and the number of scattering events with the boundaries $N_{\text {scat bound }}$ are counted during the time step. The $N_{\text {scat bound }} / N_{\mathrm{ph}}$ ratio is proportional to the scattering probability according to an exponential probability law $P_{\text {scat }}=1-\exp \left(-\delta t / \tau_{\text {scat bound }}\right)$. As long as $\delta t$ is short enough to ensure that there is at most one collision per launched phonon, thus avoiding multiple scattering, $\Lambda_{\text {scat bound }}$ MFP can be expressed as:

$$
\Lambda_{\text {scat bound }}(d, a, h)=\frac{v_{g} \delta t}{\ln \left(\frac{N_{\mathrm{ph}}}{N_{\mathrm{ph}}-N_{\mathrm{scat} \text { bound }}}\right)}
$$

Even though the phonon population depends on frequency and polarization, the boundary scattering MFP is a purely geometric quantity which only varies with the thickness of the membrane $h$, the hole diameter $d$, and the period of the lattice $a$. In case of diffusive heat conduction, the boundary scattering MFP is equal to four times volume-to-surface ratio, as explained by Blanco and Fournier [24]. In the present case, for the phononic membranes, it reads

$$
\Lambda_{\text {scat bound th }}(d, a, h)=\frac{4 V}{S}=\frac{4\left(h a^{2}-\pi h d^{2} / 4\right)}{\pi h d+2\left(a^{2}-\pi d^{2} / 4\right)}
$$

This theoretical formulation was retrieved from simulations using Eq. (3), as shown in Appendix B. The boundary scattering MFP can then be related to thermal conductivity to identify the characteristic lengths that impact heat conduction in the phononic membrane.

\section{Geometric consideration of phonon transport in phononic membranes}

Lastly, MC simulations output directional information (positions, velocities, and angles) about the phonons within the structure at each time step. Thus, ballisticity as well as directionality of heat propagation can be investigated. Here, we will present the phonon angular distribution at the end of the system for both aligned and staggered configurations to study the preferential passages.

For this purpose, velocities of phonons entering the cold reservoir are regularly recorded during the simulation, once the steady state is reached. Then the angle between their velocity vector and the $z$ axis (see Fig. 2 for axes) is computed for each phonon, and the distribution function of this "exit angle" $\theta$ is deduced.

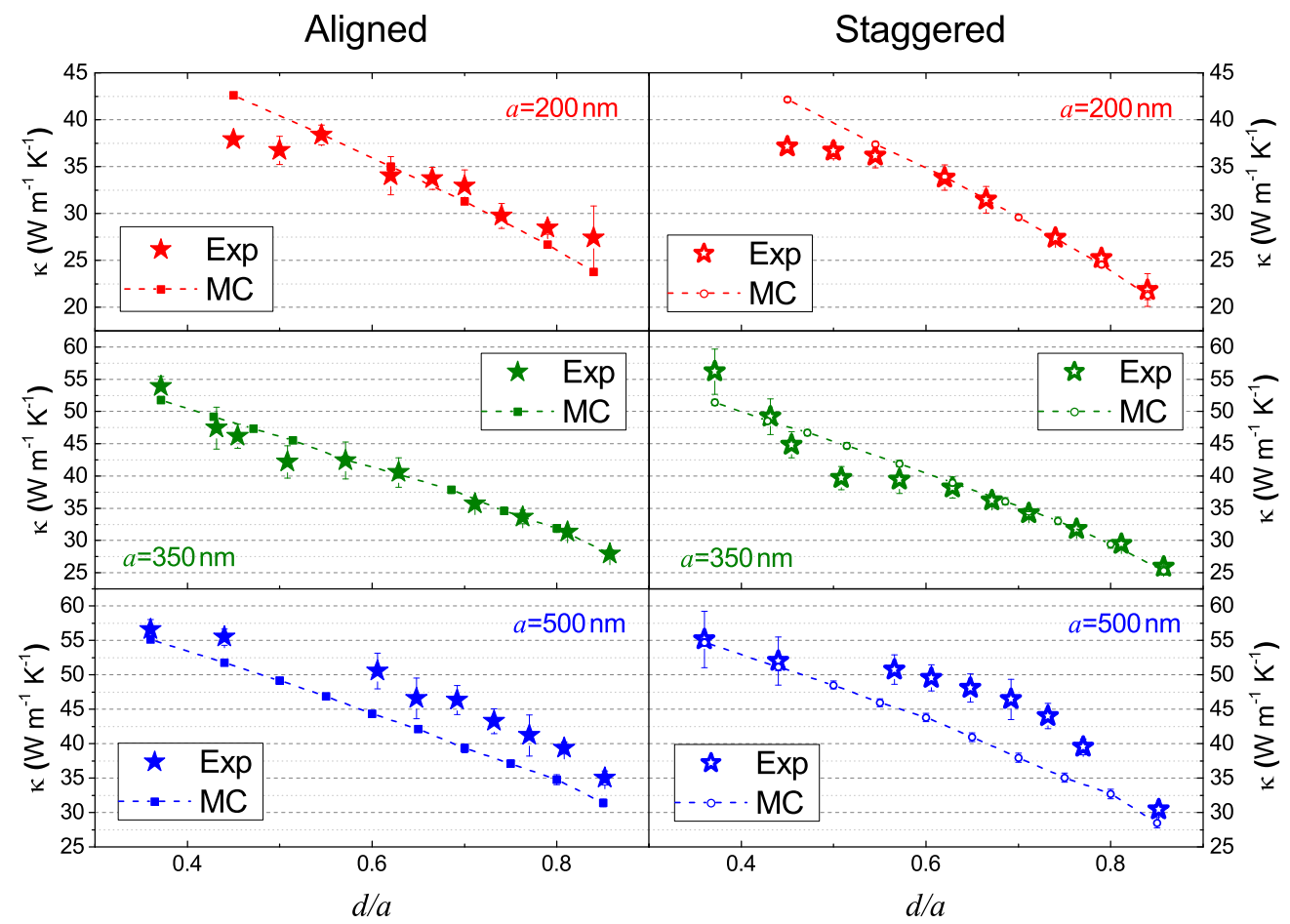

FIG. 3. Thermal conductivity of aligned and staggered phononic membranes, $\mu$-TDTR measurements, and MC simulations; $T=300 \mathrm{~K}$, $h=145 \mathrm{~nm}$; from top to bottom, $a=200,350$, and $500 \mathrm{~nm}$. Lines serve as a guide for the eyes. 


\section{THERMAL CONDUCTIVITY MEASUREMENTS AND SIMULATION RESULTS}

\section{A. Thermal conductivity at room temperature}

First, thermal conductivities extracted from $\mu$-TDTR experiments and derived from MC simulations of several phononic membranes with the same thickness of $145 \mathrm{~nm}$ are compared as a function of the $d / a$ ratio. Figure 3 shows aligned (left) and staggered (right) membranes with periods of 200, 350, and $500 \mathrm{~nm}$. The unpatterned membrane $(h=145 \mathrm{~nm})$ has in-plane thermal conductivity of $75 \mathrm{~W} \mathrm{~m}^{-1} \mathrm{~K}^{-1}$ according to experiments [8] and $68.4 \mathrm{~W} \mathrm{~m}^{-1} \mathrm{~K}^{-1}$ according to simulations. There is a reduction by a factor of two compared with bulk silicon $\left(150 \mathrm{~W} \mathrm{~m}^{-1} \mathrm{~K}^{-1}\right)$. For small periods and large $d / a$ ratios, the additional lowering of thermal conduction due to the holes can reach $70 \%$ compared with the unpatterned membrane. In both aligned and staggered cases, excellent agreement between experiments and MC simulations is observed for the periods of 350 and $200 \mathrm{~nm}$. For the period

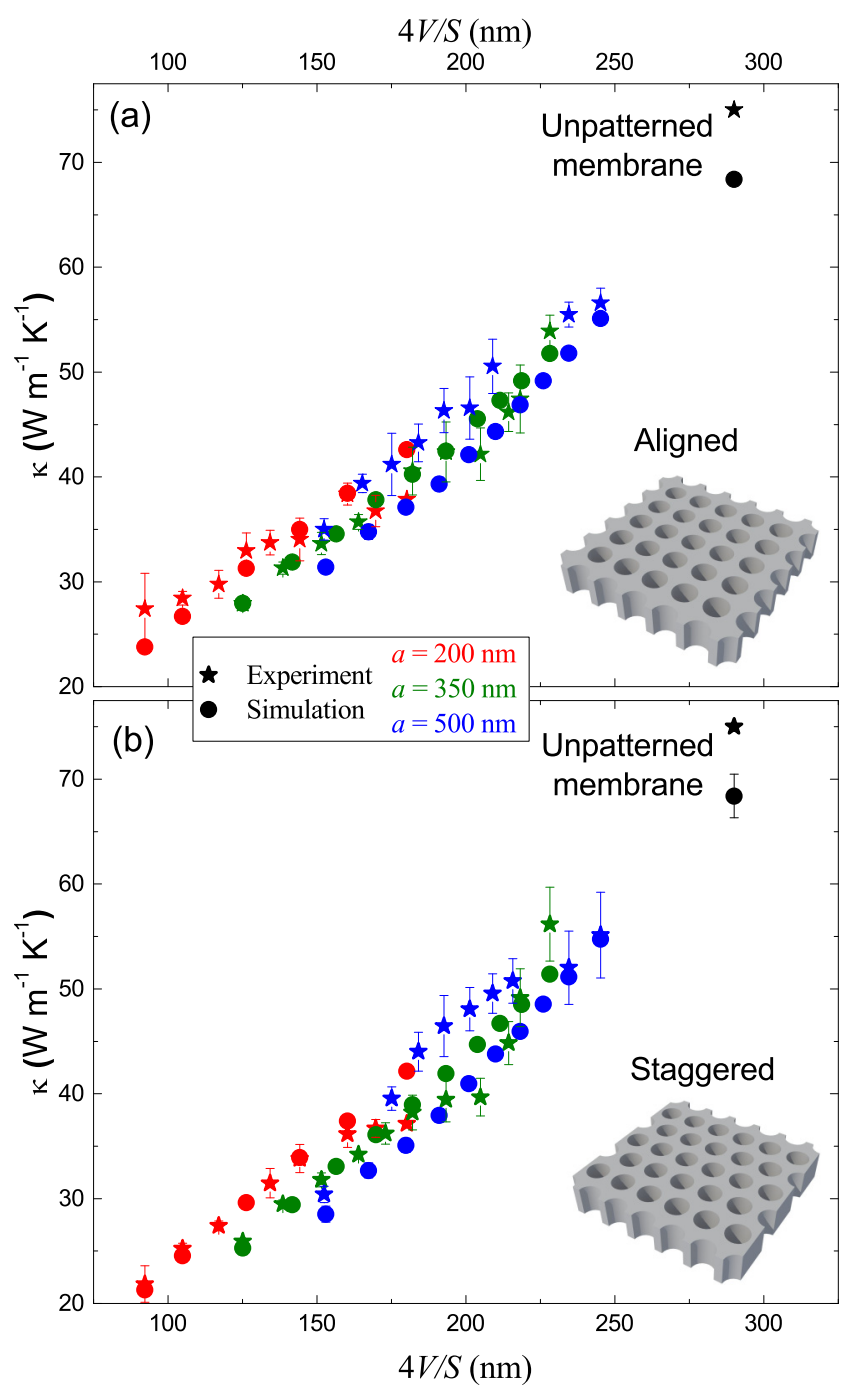

FIG. 4. Thermal conductivity of aligned and staggered phononic membranes as a function of boundary scattering MFP $\Lambda_{\text {scat bound th }}(d, a, h)=4 \mathrm{~V} / S ; \mu$-TDTR measurements and MC simulations; $T=300 \mathrm{~K}$ and $h=145 \mathrm{~nm}$. of $500 \mathrm{~nm}$, although simulations underestimate the thermal conductivity, the disagreement does not exceed 10-20\%.

For the same $d / a$, thermal conductivity is higher for longer periods $a$ in both aligned and staggered configurations. This can be understood in terms of neck size: when $a$ is large, the passage for phonons between two holes is wider. We observe a linear decrease of thermal conductivity with increasing $d / a$ ratio independent of the position of the holes (aligned or staggered). The thermal conductivity is approximately reduced twice as $d / a$ increases from 0.3 to 0.9 . For the same geometrical parameters, the staggered lattice always displays a marginally lower thermal conductivity than the aligned lattice despite the same $S / V$ ratio, as previously observed [25].

Thermal conductivity of phononic membranes can also be considered in the frame of boundary scattering MFP [Eq. (4)]. Figure 4 shows that for both lattices, experimental and theoretical data at room temperature show a clear linear trend as a function of volume-to-surface ratio, regardless of the period or the hole diameters. Computed boundary scattering MFPs are of the order of neck between holes and reach twice the thickness of the unpatterned membrane, as expected from previous studies [26]. This shows that phonon transport in those membranes is mostly diffusive due to multiple diffuse boundary scattering processes. Such a behavior was expected, as MC simulations show that temperature profile, at steady state, is linear [17]. Similar dependence of thermal conductivity on the surface-to-volume ratio, regardless of other parameters, has been demonstrated experimentally [8,27] and theoretically $[14,15]$ for various nanostructures.

\section{B. Accumulated thermal conductivity}

The accumulated thermal conductivity calculated with $\mathrm{MC}$ for bulk, unpatterned, aligned, and staggered membranes as a function of phonon intrinsic MFP [Eq. (2)] is depicted in Fig. 5. For bulk silicon, we see that most of the heat is carried by phonons with intrinsic MFPs ranging from tens to hundreds

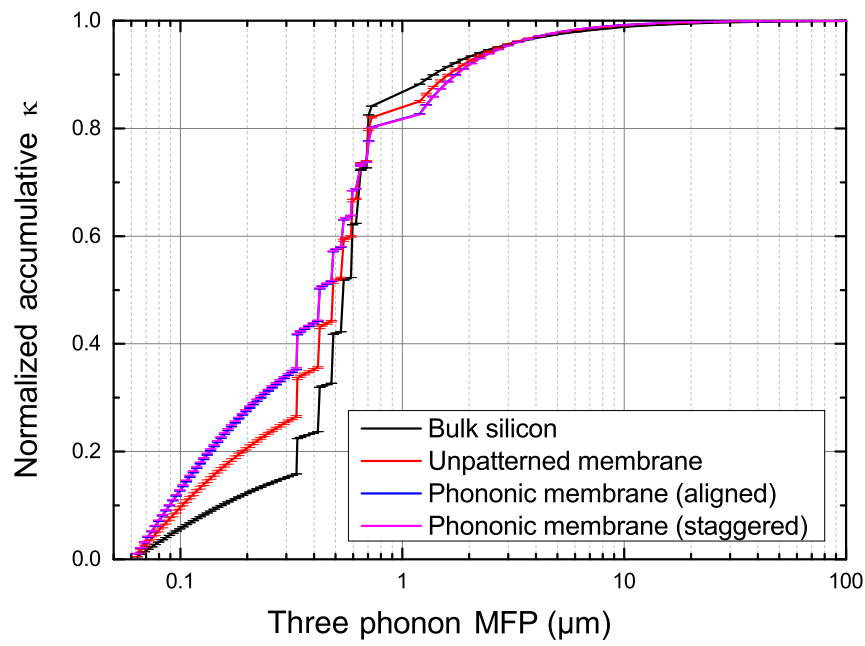

FIG. 5. Accumulated thermal conductivity of bulk and thin film and aligned and staggered phononic membranes as a function of three-phonon MFP $\Lambda$; MC simulations, $T=300 \mathrm{~K}, h=145 \mathrm{~nm}$ (for film and phononic membranes), $d=150 \mathrm{~nm}, a=200 \mathrm{~nm}$ (for aligned and staggered phononic membranes). 
of nanometers, as expected from previous studies [28]. Half of the thermal conductivity value is accumulated by phonons with intrinsic MFPs shorter than $500 \mathrm{~nm}$. This dominant three-phonon MFP becomes shorter in nanostructures, as the phonons with long intrinsic MFP are regularly scattered on membrane walls and holes. This can be understood in the frame of the phonon residence time in the simulation domain. In the case of multiple boundary scattering, a phonon does not change its state but solely its propagation direction. For phononic membranes with large $d / a$ ratio, this means that a phonon needs more time to travel from the hot to the cold reservoir. Thus, it is more likely to undergo a three-phonon scattering process. In the latter case, the state (frequency, polarization, and group velocity) of the phonon is randomly resampled according to the distribution function, and naturally, the population shifts toward TA phonons of the edge of the Brillouin zone, as expected from the phonon density of states of silicon. For example, the contribution to the thermal conductivity of phonons with intrinsic MFP shorter than $200 \mathrm{~nm}$ is only $15 \%$ for the bulk, but becomes $35 \%$ in phononic membranes. Thus, in phononic membranes, heat is mostly carried by phonons with shorter intrinsic MFP compared with bulk. However, even for a phononic membrane with $d / a=0.75$, phonons with intrinsic MFP longer than 1 $\mu \mathrm{m}$ contribute to almost $20 \%$ of the thermal conductivity. They can travel for long distances with or without boundary scattering before resetting their state and thus go through the phononic membranes.

\section{RELATIVE DIFFERENCE BETWEEN THERMAL CONDUCTIVITIES OF ALIGNED AND STAGGERED LATTICES}

\section{A. MC simulations and experiments}

The relative difference $\Delta_{\mathrm{Al}}^{\mathrm{St}}=\left(\kappa_{\mathrm{Al}}-\kappa_{\mathrm{St}}\right) / \kappa_{\mathrm{Al}}$ between thermal conductivities of aligned $\left(\kappa_{\mathrm{Al}}\right)$ and staggered $\left(\kappa_{\mathrm{St}}\right)$ configurations has been calculated for all studied geometric parameters. MC results are compared with experimental data for $T=300 \mathrm{~K}$ in Fig. 6(a). The agreement between simulations and experiments is again very good. This shows that the difference between thermal conductivities of aligned and staggered systems is well described by our MC simulations, so modifications of the dispersion relations (phononic effects) are not needed to explain the results, as the properties used in the simulations are those of the bulk.

For small values of $d / a$, both configurations have roughly the same thermal conductivity. When the $d / a$ ratio becomes larger than 0.6, the value of $\Delta_{\mathrm{Al}}^{\mathrm{St}}$ increases. Experimental points are scattered due to the additional inaccuracy generated by the FEM analysis. However, in terms of thermal decays, which are raw data of experiments, it is clear that the relative difference increases with $d / a$. MC simulations predict a difference up to $10 \%$ for large $d / a$. On the other hand, $\Delta_{\mathrm{Al}}^{\mathrm{St}}$ tends toward zero when $d / a$ is small, which is meaningful because the difference between aligned and staggered lattices of holes should disappear when the diameter of the holes goes to zero. (In both cases, the thermal conductivity tends to that of the unpatterned membrane.)

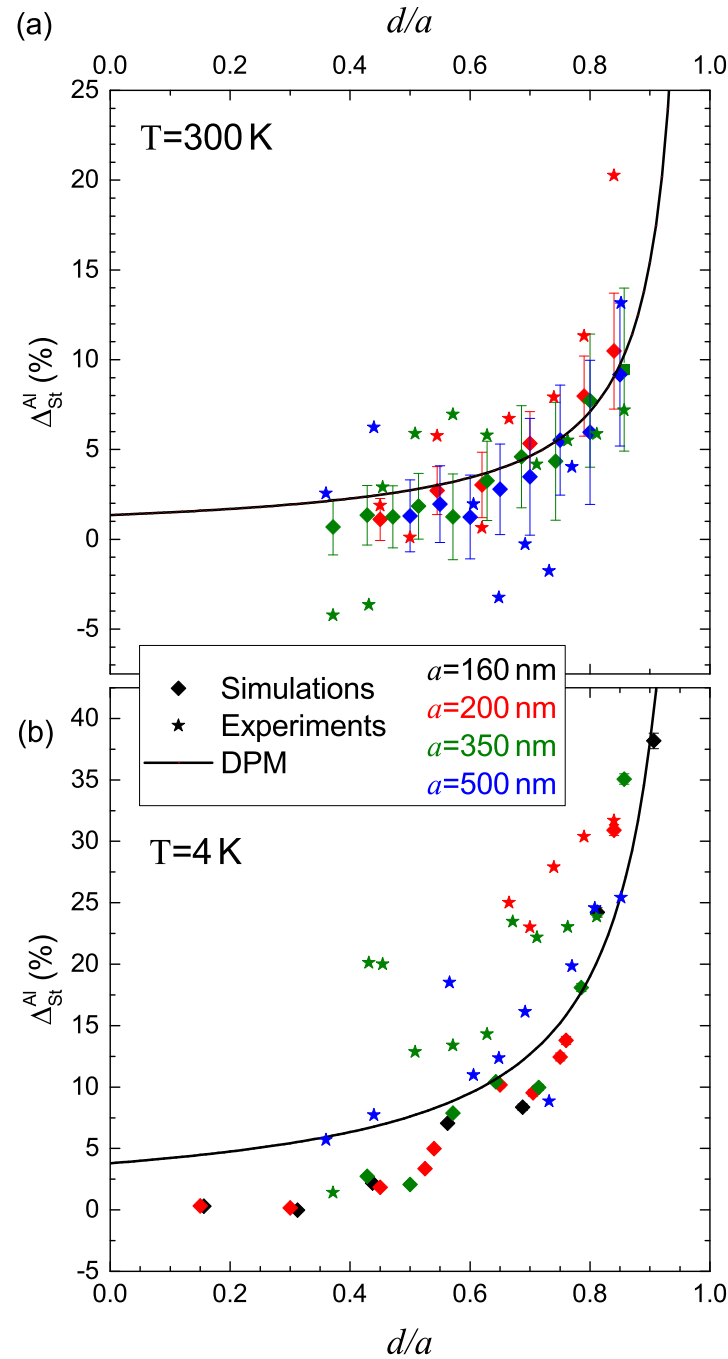

FIG. 6. Relative difference between thermal conductivities in aligned and staggered phononic membranes as a function of $d / a$ ratio at (a) $300 \mathrm{~K}$ and (b) $4 \mathrm{~K}$. Comparison between MC simulations, experiments, and DPM.

The lower thermal conductivity of staggered systems cannot be explained in terms of diffusive boundary scattering events, as the $S / V$ ratio is the same for aligned and staggered configurations and has to be explained with other considerations. In the aligned lattice, some phonons could propagate over a long distance in a straight line, passing between two rows of holes, whereas in the staggered lattice, such direct passage is at least partially obstructed by one out of two columns of holes. This hypothesis was also put forward in a previous paper [25]. If the difference between aligned and staggered lattices is really due to such directional effects, then $\Delta_{\mathrm{Al}}^{\mathrm{St}}$ should increase when the temperature is decreased as heat transport becomes more ballistic at low temperatures [29] (three-phonon MFPs are longer [30], and scattering on top and bottom surfaces of the membrane is more specular [31]). To investigate this increase of ballisticity, a similar study has been done at $T=4 \mathrm{~K}$. At this temperature, the carried energy is very small but is balanced by the very long intrinsic MFPs of phonons which are scarcely scattered by anharmonic processes [22]. 


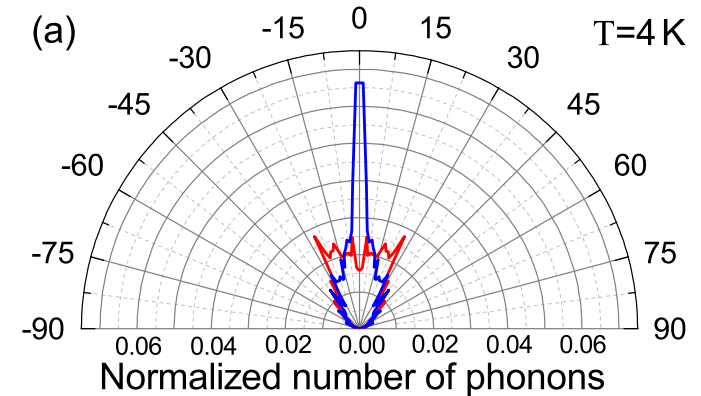

Aligned Staggered

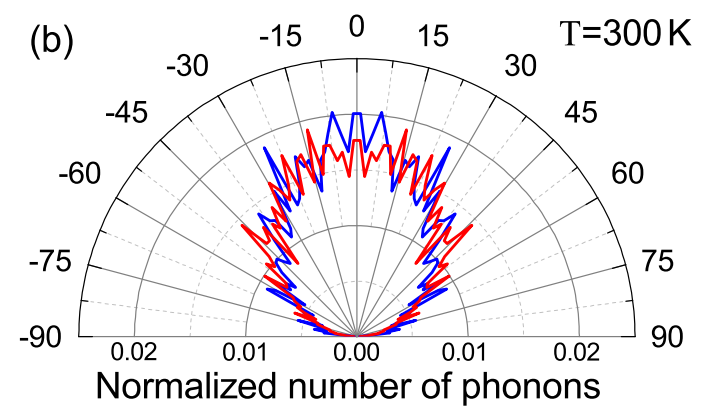

FIG. 7. Distributions of phonon exit angle $\theta\left({ }^{\circ}\right)$ at the end of the system for aligned and staggered phononic membranes $(a=$ $160 \mathrm{~nm}, d=126 \mathrm{~nm}$ ) at (a) $T=4 \mathrm{~K}$ and (b) $T=300 \mathrm{~K}$.

The relative difference between aligned and staggered lattice at $4 \mathrm{~K}$ obtained with experiments and simulations is depicted in Fig. 6(b). Even if the absolute values of thermal conductivity can be biased, the agreement on $\Delta_{\mathrm{Al}}^{\mathrm{St}}$ between simulations and measurements is acceptable. As expected from directional considerations, $\Delta_{\mathrm{Al}}^{\mathrm{St}}$ reaches larger values at $4 \mathrm{~K}$ than at $300 \mathrm{~K}$ according to both experiments and simulations. For $d / a=0.9$, MC simulations predict a relative difference between aligned and staggered lattices of almost $40 \%$ at $4 \mathrm{~K}$. Moreover, as the uncertainty of numerical results is much smaller at $4 \mathrm{~K}$ (very weak heat flux variations), we can see that $\Delta_{\mathrm{Al}}^{\mathrm{St}}$ only depends on $d / a$ and not on the period. The plot of MC data has a particular shape, with two local maxima at $d / a \sim 0.4$ and $d / a \sim 0.6$. Nevertheless it is still globally increasing with $d / a$.

To directly observe the phonons which propagate in a straight line passage between the holes in aligned lattices, the distribution of phonon angle $(\theta)$ at the end of the system for $T=4 \mathrm{~K}$ is shown in polar coordinates in Fig. 7(a) for the phononic membrane with $a=160 \mathrm{~nm}$ and $d=126 \mathrm{~nm}$. Figure 7(a) shows that most of the phonons move along the direction of measurement after passing through the aligned lattice. More than $80 \%$ of these phonons are located in front of the neck, which means that they surely propagated ballistically between the holes. In the staggered lattice, there is no observable peak around $0^{\circ}$ because these phonons have been scattered by the holes and their direction has changed. However, despite the fluctuations, the angle distribution for the staggered lattice shows that more phonons exit the system with angles around $-25^{\circ}$ and $25^{\circ}$ that correspond to the straight

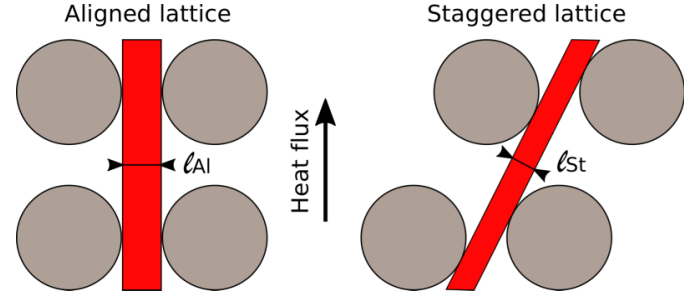

FIG. 8. Direct passage between the holes for the aligned and staggered lattices.

directions between the holes in this case (see the Direct Passage Model section).

However, at $T=300 \mathrm{~K}$ the distributions for aligned and staggered lattices were broader and roughly the same [Fig. 7(b)]. No directionality was observed due to statistical uncertainties and scattering (anharmonic processes and scattering by the membrane's top and bottom surfaces, which are not specular at room temperature). Nevertheless, phonons with long intrinsic MFP still have a better chance to exit the system at $90^{\circ}$ in an aligned lattice than in a staggered lattice.

\section{B. Direct passage model}

We have established that phonons exiting the phononic membrane have rather specific directions following the angles defined by the direct passages between the holes. Here, we develop a simple model, called Direct Passage Model (DPM), which takes into account the width of the direct passages in the aligned and staggered lattices to explain the difference in thermal conductivity between these two lattices.

In the DPM, we start by assuming that the thermal conductivities of the aligned lattice and staggered lattice are identically proportional to the width of the direct passage $\ell$ : $\kappa \propto \ell^{\alpha}$, where $\alpha$ is determined by experiments or simulations (Appendix C). In the case of the aligned (Al) lattice of holes, the width of the direct passage is simply equal to the neck $\ell_{\mathrm{Al}}=n=a-d$, as depicted in Fig. 8. For the staggered $(\mathrm{St})$ lattice, the size of the direct passage $\ell_{\mathrm{St}}$ is smaller than $\ell_{\mathrm{Al}}$ for the same period and diameter and equal to $\ell_{\mathrm{St}}=2 a / \sqrt{5}-d$. Because of the symmetry, a second passage of width $\ell_{\mathrm{St}}$ should be considered in the staggered lattice, but the heat flux split in two in these two passages. Thus, we can consider only one passage of width $\ell_{\mathrm{St}}$ for the total heat flux.

At this stage, one can quickly see that $\ell_{\mathrm{St}}=0$ yields $\kappa_{\mathrm{St}}=0$, which is obviously incorrect because in that case the neck is $n_{\mathrm{St}, 0}=a(1-2 / \sqrt{5})$, which is not zero. Actually, $\ell_{\mathrm{St}}=0$ means that ballistic phonons must necessarily collide on the holes of the staggered lattice. Because of the large number of collisions, the phonons in this situation can be considered diffused. By analogy with the Eucken model, we assume a correction factor for the porosity $n_{\mathrm{St}, 0}\left(\phi_{\mathrm{St}, 0} / \phi_{n, 0}\right)^{2}$, where $\phi_{\mathrm{St}, 0}=\pi / 5$ is the porosity for $\ell_{\mathrm{St}}=0$ and $\phi_{n, 0}=\pi / 4$ is the porosity for $n=0$.

The final expressions of the thermal conductivities for the aligned and staggered lattices in the DPM are 
given by:

$$
\begin{gathered}
\kappa_{\mathrm{Al}} \propto\left(\ell_{\mathrm{Al}}\right)^{\alpha}=n^{\alpha} \\
\kappa_{\mathrm{St}} \propto\left[\ell_{\mathrm{St}}+n_{\mathrm{St}, 0}\left(\frac{\phi_{\mathrm{St}, 0}}{\phi_{n, 0}}\right)^{2}\right]^{\alpha} \\
=\left[\frac{2 a}{\sqrt{5}}-d+a\left(1-\frac{2}{\sqrt{5}}\right) \frac{\phi_{\mathrm{St}, 0}^{2}}{\phi_{n, 0}^{2}}\right]^{\alpha}
\end{gathered}
$$

where the proportionality factor cancels when we compute the relative difference $\Delta_{\mathrm{Al}}^{\mathrm{St}}$. Also, by factorizing with $a$, we see that $\Delta_{\mathrm{Al}}^{\mathrm{St}}$ becomes dependent only on the $d / a$ ratio:

$$
\Delta_{\mathrm{Al}}^{\mathrm{St}}=1-\left[\frac{\frac{2}{\sqrt{5}}-\frac{d}{a}+(1-2 / \sqrt{5}) \phi_{\mathrm{St}, 0}^{2} / \phi_{n, 0}^{2}}{1-d / a}\right]^{\alpha}
$$

At $300 \mathrm{~K}$, the thermal conductivity follows a power law $\kappa \propto n^{0.35}$, whereas at $4 \mathrm{~K}$, it can be approximated by $\kappa \propto n$; thus, $\alpha$ is equal to 0.35 at $300 \mathrm{~K}$ and to 1 at $4 \mathrm{~K}$ (the determination of $\alpha$ is done in Appendix C). Figure 6(a) shows good agreement between the model, experimental data, and MC simulation results at both 4 and $300 \mathrm{~K}$.

The model predicts that the relative difference at $4 \mathrm{~K}$ is larger than at $300 \mathrm{~K}$, as we would expect because of the decreased scattering rate. The DPM also successfully explains the superposition of the points as a function of $d / a$, as well as the period dependence when $\Delta_{\mathrm{Al}}^{\mathrm{St}}$ is plotted as a function of the neck (see details in Appendix D). Interestingly, the MC simulations show two local maxima located at $d / a=$ 0.4 and 0.6 , which are not captured by the DPM. One possible explanation is that, in this state, the DPM takes into account only the widest direct passage, whereas several smaller passages actually exist for $d / a<2 / \sqrt{5}$. However, the integration of these other passages is nontrivial and requires further investigations.

\section{CONCLUSION}

In this paper, we investigated how the thermal conductivity of phononic membranes is affected by geometric parameters, such as the period, the diameter of the holes, the $S / V$ ratio, and the pattern of the hole lattice. Comparing thermal conduction in aligned and staggered lattices of holes, MC simulations of phonon transport based on bulk properties (dispersion curves, phonon lifetimes, etc.) quantitatively reproduced experimental results at $300 \mathrm{~K}$ without any adjustment parameters.

The present paper also demonstrates that the difference between thermal conductivity of aligned and staggered lattices arises from directional effects when considering phonon transport. Indeed, hole occurrence induces strong backscattering of phonons and thus naturally reduces thermal conductivity. Nevertheless, at room temperature, MFP spectral analyses reveal some evidence that even phonons with long MFPs still contribute to thermal conductivity. To understand this behavior, we can assume that a part of heat conduction in phononic membranes is ballistic, as low frequency phonons can travel long distances (greater than the period or the neck) between holes, even if they are subject to boundary scattering. Such directional effects could not be observed in phononic
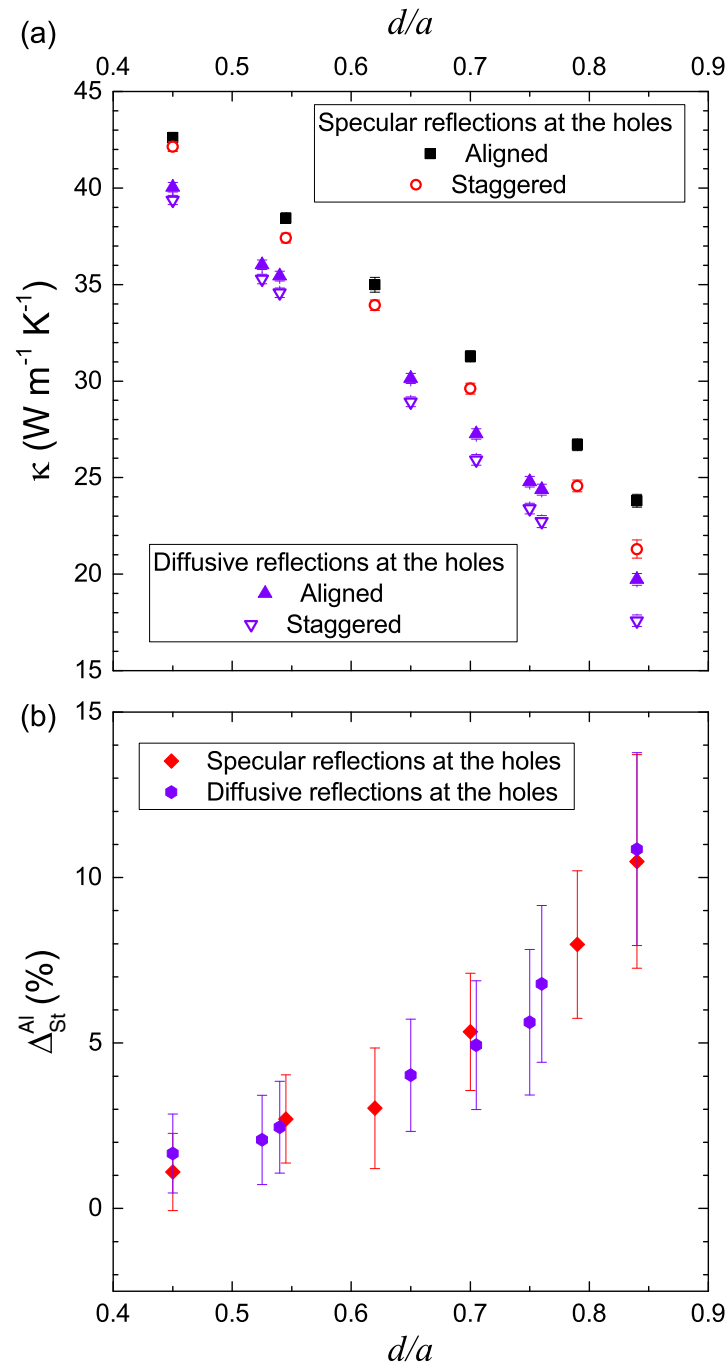

FIG. 9. Comparison of specular and diffusive reflections of phonons at the holes for $a=200 \mathrm{~nm}$ at $T=300 \mathrm{~K}$. (a) Thermal conductivity of aligned and staggered lattices. (b) Relative difference between thermal conductivity of aligned and staggered lattices.

membranes with macroscopic characteristic lengths [32], as three-phonon scattering processes will dominate, as in bulk materials.

At low temperature, heat conduction is partly ballistic (phonon intrinsic MFPs are longer), so the difference between aligned and staggered lattices increases, which allowed us to directly observe ballistic phonons with MC simulations. Thus, designing the lattice of phononic membranes could help to control the propagation direction of phonons. One can imagine other lattices than aligned and staggered which could focus or spread phonons in specific regions.

Finally, we developed a simple model that explains thermal conduction in phononic membranes with both lattices for different $d / a$ ratios at $4 \mathrm{~K}$ and $300 \mathrm{~K}$. This model, based only on geometric considerations (straight passages between holes), can predict the difference in thermal conductivity between the two lattices, and its accuracy could be improved by taking into account more straight passages. A further study could also investigate the effect of the lattice on thermal conductivity for 
a wide range of temperatures in order to fully understand the transition from ballistic to diffusive heat conduction regimes.

\section{ACKNOWLEDGMENTS}

The authors acknowledge Prof. M. Nomura (Tokyo University) for useful discussions and support throughout this study. Calculations were partly performed on the large computer facilities Grand Equipement National de Calcul Intensif-Institut de Développement et des Ressources en Informatique Scientifique (GENCI-IDRIS) (Grant No. 2014-x2014097186) and on the Ermione cluster (Institut Jean Lamour (IJL)-LEMTA). This work was supported by Postdoctoral Fellowship of Japan Society for the Promotion of Science. They also thank Dr. J. Maire for assistance in sample fabrication and data analysis.

\section{APPENDIX A}

At first glance, specular boundary condition at the holes was assumed for Monte Carlo simulations at room temperature in order to maximize the possible impact of the hole pattern on ballistic transport. Then, the impact of the hole boundary condition (specular or diffuse reflections) on thermal conductivity in Monte Carlo simulations was investigated for one period ( $a=200 \mathrm{~nm}$ ). It was found that, at room temperature, the diffuse condition just slightly reduced the thermal conductivity compared with the specular condition [Fig. 9(a)]. When the holes became large, the difference between thermal conductivities of membranes with specular and diffuse reflections at the holes increased a bit, as the neck was strongly reduced. Yet it remained less than $15 \%$.

In fact, diffuse or specular reflections at a boundary really changes thermal conductivity only when the boundary is parallel to the heat flux. In this case, only diffuse condition will lead to possible backscattering of phonons and reduction

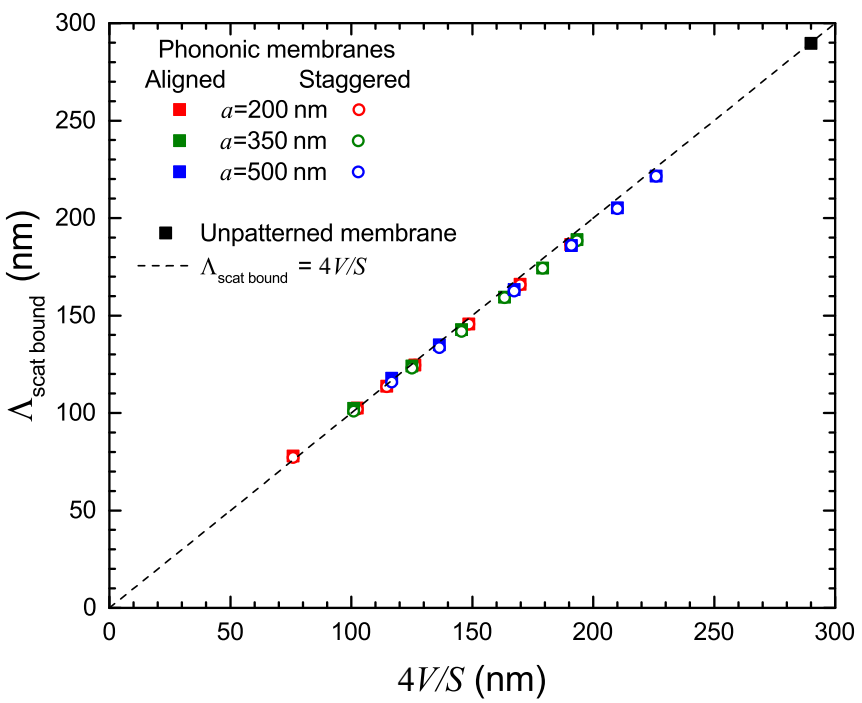

FIG. 10. Boundary scattering MFP obtained from MC ray tracing simulations as a function of theoretical boundary scattering MFP [Eq. (4)], which is equal to $4 \mathrm{~V} / \mathrm{S}$, for the unpatterned membrane $(h=145 \mathrm{~nm})$ and aligned and staggered phononic membranes. The symbols for aligned systems are hidden by those for staggered systems because both lattices have the same boundary scattering MFP. of thermal conductivity. This is why diffuse conditions are mandatory on membrane walls at room temperature. When the boundary is partly perpendicular to the heat flux, though, as for the holes in phononic membranes, the specular or diffuse condition has less impact on global thermal transport because even specular reflections can lead to backscattering.

Moreover, it is verified from Fig. 9(b) that the relative difference between aligned and staggered lattices is the same for both diffuse and specular conditions at the holes. As the impact of the hole boundary condition on thermal transport and directionality is found to be weak, simulations with specular reflections were kept.

\section{APPENDIX B}

The boundary scattering MFP has been computed from $\mathrm{MC}$ ray tracing simulations using Eq. (3). The computed $\Lambda_{\text {scat bound }}$ for the unpatterned membrane $(h=145 \mathrm{~nm})$ and aligned and staggered phononic membranes with different periods is plotted as a function of the theoretical boundary scattering MFP [Eq. (4)] in Fig. 10. All the points follow the
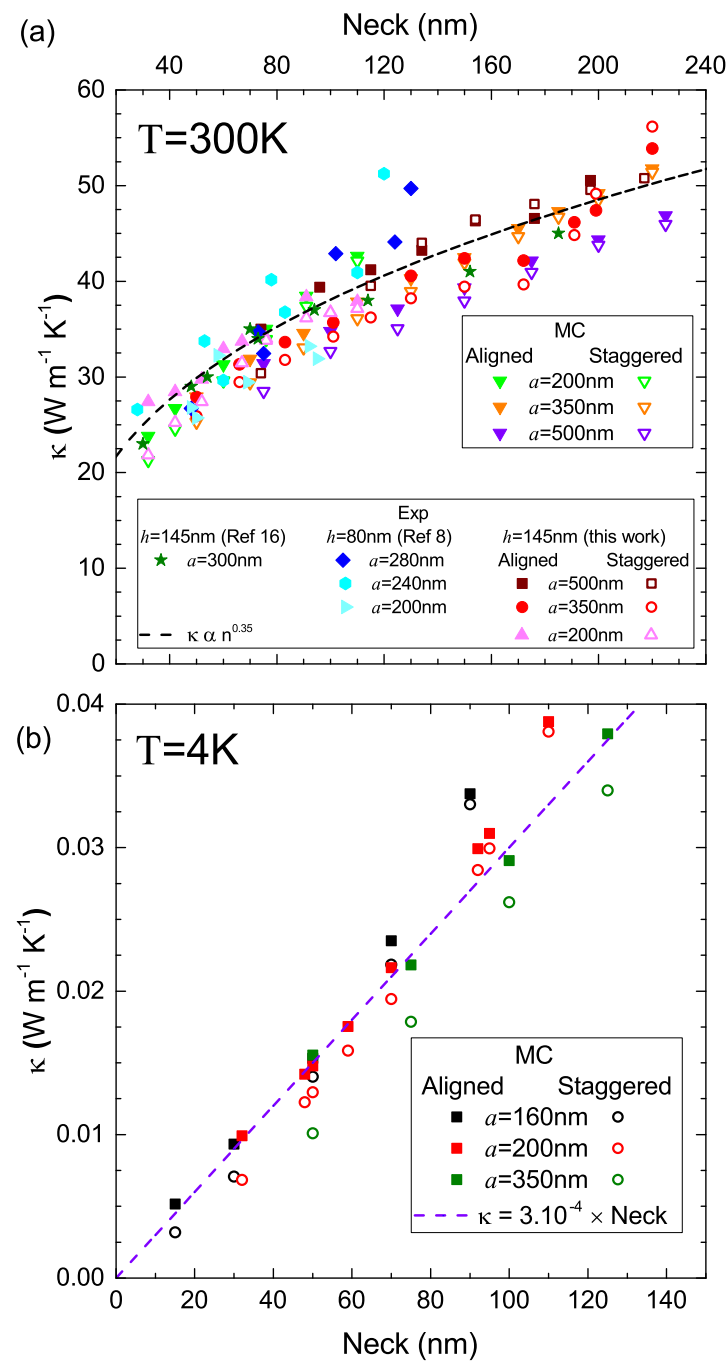

FIG. 11. Power law approximation of thermal conductivity as a function of the neck for aligned and staggered lattices of different periods at (a) $300 \mathrm{~K}$ and (b) $4 \mathrm{~K}$. 
theoretical plot $\Lambda_{\text {scat bound }}=4 \mathrm{~V} / \mathrm{S}$. This proves the validity of Eq. (4) for unpatterned and phononic membranes.

\section{APPENDIX C}

In order to determine the power $\alpha$ for the DPM, we had to fit the thermal conductivity of phononic membranes. Figures 11(a) and 11(b) show the fits of thermal conductivities obtained in this paper and found in the literature $[8,16]$ at 300 and $4 \mathrm{~K}$, respectively, as a function of the neck $\left(\ell_{\mathrm{Al}}\right)$. At $300 \mathrm{~K}$, the same approximation with $\alpha=0.35$ is able to describe both simulations and experimental results (experimental data are taken from various references) for aligned and staggered lattices. At $4 \mathrm{~K}$, the thermal conductivity can be well approximated by a linear fit, as long as the neck is small compared with the period. Only MC results are plotted in Fig. 11(b), but the experimental data at $4 \mathrm{~K}$ also follow a linear trend. We deduce that $\alpha=1$ at $4 \mathrm{~K}$.

\section{APPENDIX D}

At $4 \mathrm{~K}$, plotting $\Delta_{\mathrm{Al}}^{\mathrm{St}}$ values obtained with $\mathrm{MC}$ as a function of the neck shows a period dependence (Fig. 12). For the same neck, $\Delta_{\mathrm{Al}}^{\mathrm{St}}$ is larger for large periods. Figure 12 shows that

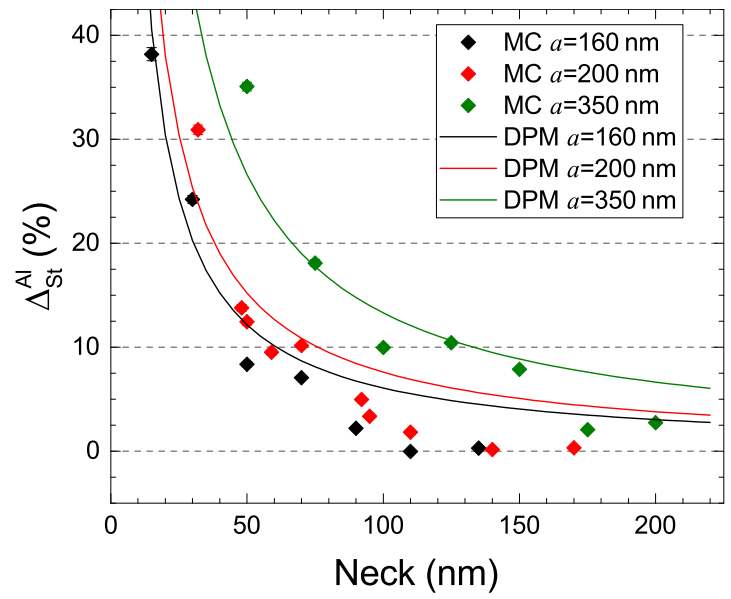

FIG. 12. Relative difference between aligned and staggered lattice thermal conductivities at $4 \mathrm{~K}$ as a function of the neck, comparison between MC simulations, and the DPM.

the DPM correctly predicts that a larger period yields a larger difference between the thermal conductivities of the aligned and staggered lattices.
[1] D. G. Cahill, P. V. Braun, G. Chen, D. R. Clarke, S. Fan, K. E. Goodson, P. Keblinski, W. P. King, G. D. Mahan, A. Majumdar, H. J. Maris, S. R. Phillpot, E. Pop, and L. Shi, Appl. Phys. Rev. 1, 11305 (2014).

[2] Z. Wei, J. Yang, K. Bi, and Y. Chen, J. Appl. Phys. 118, 155103 (2015).

[3] E. Chavez-Angel, J. S. Reparaz, J. Gomis-Bresco, M. R. Wagner, J. Cuffe, B. Graczykowski, A. Shchepetov, H. Jiang, M. Prunnila, J. Ahopelto, F. Alzina, and C. M. Sotomayor Torres, APL Mater. 2, 012113 (2014).

[4] D. Li, Y. Wu, P. Kim, L. Shi, P. Yang, and A. Majumdar, Appl. Phys. Lett. 83, 2934 (2003).

[5] A. L. Moore, S. K. Saha, R. S. Prasher, and L. Shi, Appl. Phys. Lett. 93, 083112 (2008).

[6] R. H. Tarkhanyan and D. G. Niarchos, Int. J. Therm. Sci. 67, 107 (2013).

[7] R. Dettori, C. Melis, X. Cartoixa, R. Rurali, and L. Colombo, Phys. Rev. B 91, 054305 (2015).

[8] R. Anufriev, J. Maire, and M. Nomura, Phys. Rev. B 93, 045411 (2016).

[9] J. Tang, H. T. Wang, D. H. Lee, M. Fardy, Z. Huo, T. P. Russell, and P. Yang, Nano Lett. 10, 4279 (2010).

[10] J. Lim, H.-T. Wang, J. Tang, S. C. Andrews, H. So, J. Lee, D. H. Lee, T. P. Russell, and P. Yang, ACS Nano 10, 124 (2016).

[11] J.-K. Yu, S. Mitrovic, D. Tham, J. Varghese, and J. R. Heath, Nat. Nanotechnol. 5, 718 (2010).

[12] N. K. Ravichandran and A. J. Minnich, Phys. Rev. B 89, 205432 (2014).

[13] A. Jain, Y. J. Yu, and A. J. H. McGaughey, Phys. Rev. B 87, 195301 (2013).

[14] M. Verdier, K. Termentzidis, and D. Lacroix, J. Appl. Phys. 119, 175104 (2016).
[15] M.-S. Jeng, R. Yang, D. Song, and G. Chen, J. Heat Transfer 130, 42410 (2008)

[16] M. Nomura, Y. Kage, J. Nakagawa, T. Hori, J. Maire, J. Shiomi, R. Anufriev, D. Moser, and O. Paul, Phys. Rev. B 91, 205422 (2015).

[17] D. Lacroix, K. Joulain, and D. Lemonnier, Phys. Rev. B 72 , 064305 (2005).

[18] J. P. M. Péraud and N. G. Hadjiconstantinou, Phys. Rev. B 84, 205331 (2011).

[19] V. Jean, S. Fumeron, K. Termentzidis, X. Zianni, and D. Lacroix, Int. J. Heat Mass Transfer 86, 648 (2015).

[20] E. Pop, R. W. Dutton, and K. E. Goodson, J. Appl. Phys. 96, 4998 (2004).

[21] M. G. Holland, Phys. Rev. 134, A471 (1964).

[22] M. G. Holland, Phys. Rev. 132, 2461 (1963).

[23] V. Jean, S. Fumeron, K. Termentzidis, S. Tutashkonko, and D. Lacroix, J. Appl. Phys. 115, 24304 (2014).

[24] S. Blanco and R. Fournier, Europhys. Lett. 61, 168 (2003).

[25] R. Anufriev, A. Ramiere, J. Maire, and M. Nomura, Nat. Commun. 8, 15505 (2017).

[26] Y. F. Zhu, J. S. Lian, and Q. Jiang, Appl. Phys. Lett. 92, 113101 (2008).

[27] L. Yang, Y. Yang, Q. Zhang, Y. Zhang, Y. Jiang, Z. Guan, M. Gerboth, J. Yang, Y. Chen, D. Greg Walker, T. T. Xu, and D. Li, Nanoscale 8, 17895 (2016).

[28] A. Minnich and G. Chen, Appl. Phys. Lett. 91, 73105 (2007).

[29] J. Maire, R. Anufriev, and M. Nomura, Sci. Rep. 7, 41794 (2017).

[30] L. Weber and E. Gmelin, Appl. Phys. A Mater. Sci. Process. 53, 136 (1991).

[31] M. Asheghi, Y. K. Leung, S. S. Wong, and K. E. Goodson, Appl. Phys. Lett. 71, 1798 (1997).

[32] D. Song and G. Chen, Appl. Phys. Lett. 84, 687 (2004). 